\title{
Lead, Manganese, and Methylmercury as Risk Factors for Neurobehavioral Impairment in Advanced Age
}

\author{
Bernard Weiss \\ Department of Environmental Medicine, School of Medicine and Dentistry, University of Rochester, Rochester, NY 14642, USA \\ Correspondence should be addressed to Bernard Weiss, bernard_weiss@urmc.rochester.edu
}

Received 13 September 2010; Revised 28 October 2010; Accepted 24 November 2010

Academic Editor: Peter Faller

Copyright () 2011 Bernard Weiss. This is an open access article distributed under the Creative Commons Attribution License, which permits unrestricted use, distribution, and reproduction in any medium, provided the original work is properly cited.

Contamination of the environment by metals is recognized as a threat to health. One of their targets is the brain, and the adverse functional effects they induce are reflected by neurobehavioral assessments. Lead, manganese, and methylmercury are the metal contaminants linked most comprehensively to such disorders. Because many of these adverse effects can appear later in life, clues to the role of metals as risk factors for neurodegenerative disorders should be sought in the exposure histories of aging populations. A review of the available literature offers evidence that all three metals can produce, in advanced age, manifestations of neurobehavioral dysfunction associated with neurodegenerative disease. Among the critical unresolved questions is timing; that is, during which periods of the lifespan, including early development, do environmental exposures lay the foundations for their ultimate effects?

\section{Introduction}

In soliciting contributions for this special issue of the International Journal of Alzheimer's Disease, the editors featured the metals copper, zinc, iron, and aluminum. Except perhaps for aluminum, none of the four are considered to be major environmental hazards for neurodegenerative disorders. The three metals addressed in this paper provide a contrasting view. Lead, organic mercury, and manganese are identified as environmental risk factors for such disorders because of the scale of their distribution. It is worth reviewing how they achieved this status because such an appraisal may offer a useful model for the ways in which we evaluate the heath risks of other metals as well as those discussed here.

Environmental neurotoxicology adopted a view of the coupling of metal exposure and disease determined by its early connections to risk assessment. It approached its evaluations from angles dictated by legislation and regulation, especially after passage, by the US Congress, of the Toxic Substances Control Act (TSCA), signed into law in 1976.

As practiced by the US Environmental Protection Agency, and, in fact, by its counterparts in other nations, its primary task is to determine the health risks of exposure to environmental agents, quantify them, and then prescribe exposure standards that offer an adequate margin of safety. Although economic impacts are to be considered, typically, because exposures to environmental chemicals offer no health benefits, the health risks assume priority and exposure standards are sought that offer a robust margin of safety. The standards are not formulated specifically to protect against clinical disorders such as Alzheimer's disease. Instead, the margin of safety is designed to provide a buffer against even minimal adverse effects. The exposure criterion is designated as the Reference Dose (RfD) and is defined as "An estimate, with uncertainty spanning perhaps an order of magnitude, of a daily oral exposure to the human population (including sensitive subgroups) that is likely to be without an appreciable risk of deleterious effects during a lifetime." This definition is pertinent to disease etiology because it underscores the developing consensus that neurodegenerative disorders may originate in events and circumstances that antedate, even by decades, their overt clinical manifestations. Further, the first indications of such a progression may take the form of subtle, insidious, minimal toxic manifestations that foreshadow the emergence of clinically apparent disorders [1].

Long before the concept of environmental protection arose, along with its regulatory machinery, all three metals 
Table 1: Age-specific incidence rates for Alzheimer's disease. Mean estimates are the averages of the smoothed age-specific incidence curves from these studies [2].

\begin{tabular}{lc}
\hline Age, years & Estimate \\
\hline 60 & 0.08 \\
65 & 0.17 \\
70 & 0.35 \\
75 & 0.71 \\
80 & 1.44 \\
85 & 2.92 \\
90 & 5.95 \\
95 & 12.10 \\
\hline
\end{tabular}

had been identified as neurotoxicants. Lead and mercury had been acknowledged as poisons even in ancient texts. Manganese came to be viewed as a neurological poison in the 19th century. The new principles of environmental protection changed the equation to a focus on early detection of potential adverse effects.

The connection between the principles and practices of environmental protection and the role of metals in neurodegenerative disorders may seem superficially remote. It is, in fact, one model for how we might formulate research on that question and how to convert that research into practice. That model emphasizes the early stages of the progression to a clinical entity.

The perspective adopted by this paper adheres to that approach. One way in which it might be depicted graphically appears in Figure 1. It was formulated originally [3] to show how even a small shift in the IQ distribution of a population exposed to elevated levels of lead can create disproportionate consequences at the extremes of the distribution. As shown there, a $5 \%$ lowering of the mean (5 IQ points on standardized tests) leads to a $57 \%$ increase in the proportion of the population classified as "retarded," a definition based on the usual criterion of an IQ of 70. With a mean of 100, the usual standardized mean, and a standard deviation (SD) of 15, as on the Stanford-Binet, an IQ of 70 lies two SDs below the mean.

Figure 1 is only a model for how to depict a specific measure. A similar chart could be made for a component of cognitive function such as memory. On a population basis, most aspects of cognitive function decline with age. According to the analysis of Hedden and Gabrieli [4], these include inductive reasoning, spatial orientation, perceptual speed, and verbal memory. Vocabulary remains intact or improves. Drag and Bieliauskas [5] also noted that vocabulary remains resistant to aging and may even improve, but that both longterm and short-term memory and processing speed decline. All show distributions of scores that fit the model in Figure 1.

Assume, provisionally, that for a selected age group, we plot a distribution of an ability, such as memory, as in Figure 1. With progressively older age groups, the mean of the distribution will shift to lower scores, which means that an increasingly greater proportion of that population will fall within what we might define as a zone of impairment.
We might define such a zone as that proportion two SDs or more below the mean of a criterion group such as adults 25-45 years of age. With a typical normal or bell-shaped distribution, the older the age group is, the greater will be the impact of a specific shift in the mean, such as one caused by an environmental exposure because a greater total percentage of the age group will fall into the impairment zone. Put another way, the exposure is not a direct cause of the disease; rather, it shifts the population's cognitive performance toward scores that are characteristic of an older population.

Neurodegenerative diseases follow a pattern, as exemplified by Alzheimer's disease (AD), in which prevalence increases with age. Brookmeyer et al. [2] calculated agespecific incidence rates for $\mathrm{AD}$ from a body of data provided by four studies. Their mean estimates are the averages of the smoothed age-specific incidence curves from these studies. Their predicted percentages of cases per age group appear in Table 1. Hebert et al. [6], based on their studies of three Chicago populations, show striking changes in the proportions of individuals within three age groups (65$74,75-84$, and $>85$ ) in the scores attained on the MiniMental State Examination (MMSE), with a sharp rise in the severe category in patients over 85 years of age. Thus, both diminished cognitive function and $\mathrm{AD}$ follow an equivalent age pattern. Were MMSE score distributions to be shifted downward in an exposed population by, say, 5\%, we could legitimately interpret the exposure as a risk factor for $\mathrm{AD}$.

In the following discussions of lead, manganese, and mercury I will review data indicating that, at environmentally relevant levels, such exposures augment the expected decline in neurobehavioral function associated with aging. I will use these data to argue that such exposures then constitute risk factors for neurodegenerative disease states. That is, they would not be labeled as the primary sources of these diseases. Rather, (1) they elevate the likelihood that latent factors, such as genetic predispositions, will find overt expression or (2) they cause the clinical form of the disease to appear earlier in the lifespan. For example, the onset of clinically diagnosed $\mathrm{AD}$ is often preceded by a transitional phase termed mild cognitive impairment (MCI). It describes a condition in which neuropsychological function, particularly memory, is compromised, but not enough to meet the criteria for a clinical diagnosis of $\mathrm{AD}$, recognizing that the ultimate criterion for such a diagnosis is neuropathology. If an environmental exposure itself is able to magnify MCI, it would be considered a risk factor for clinically manifested AD as well as a condition that diminishes the individual's quality of life.

\section{Lead}

Although identified as a neurotoxicant as long ago as the second century BCE, its effects on neurobehavioral development have generated the greatest volume of research during the past five decades. Effects on adults occupy only a small proportion of the contemporary literature, and effects during advanced age an even smaller proportion. 


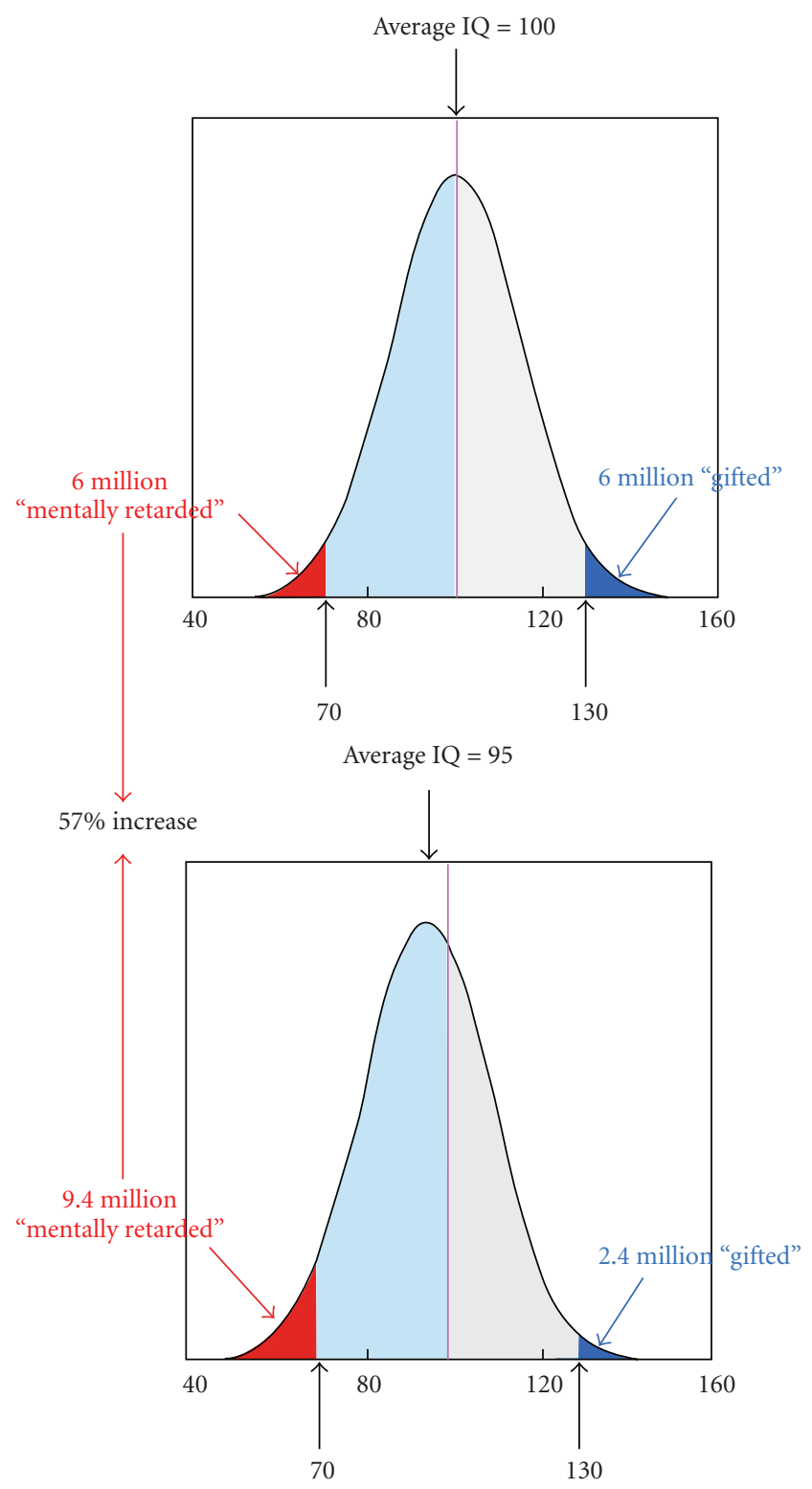

FIGURE 1: Showing the consequences of a shift in the population IQ of 5 points $(5 \%)$ in a population of 100 million. In most US school districts, an IQ of 70 or lower (2 SDs below the mean) mandates remedial action. (Based on Weiss [3]).

That imbalance is slowly undergoing a shift, one propelled by increasing recognition of the health risks posed to a ballooning population of the elderly. Among the risks arousing the greatest concerns are those bearing on cognitive function. Other clinical entities such as osteoporosis and hypertension can be treated with drugs, which is a useful property given that lead is a risk factor for hypertension, coupled with the connections between hypertension and multi-infarct dementia as well as with cognitive decline. Cognitive dysfunction itself, at this stage of knowledge, lies mostly outside the realm of pharmacotherapy.

The US Centers for Disease Control and Prevention (CDC) has set a value of 10 micrograms of lead per deciliter
TABLE 2: Signs of manganese poisoning.
(i) Impaired coordination
(ii) Abnormal gait
(iii) Abnormal laughter
(iv) Expressionless face
(v) Weakness
(vi) Bradykinesia
(vii) Somnolence
(viii) Dysarthria
(ix) Difficulty walking
(x) Clumsiness
(xi) Lack of balance
(xii) Muscle pains
(xiii) Diminished leg power

of blood $(\mu \mathrm{g} / \mathrm{dL})$ as the level at which it recommends that public health actions be initiated for young children because of the recognized vulnerability of the developing brain. This value is the latest in a progression of exposure criteria that have evolved in parallel with accumulating information about the scope of lead neurotoxicity and the low levels at which neurotoxicity is observed. Gilbert and Weiss [7], however, have argued that this value is too high and that $2 \mu \mathrm{g} / \mathrm{dL}$ is a more reasonable figure given that no discernible threshold for lead toxicity has been established.

What can be gleaned from the current lead literature is that older adults appear to be as vulnerable as children to lead neurotoxicity. Muldoon et al. [8] measured neuropsychological function in a cohort of elderly women and found that levels as low as $8 \mu \mathrm{g} / \mathrm{dL}$ were significantly associated with poorer cognitive function. As they noted, "even a slight decrement in cognition would have a large public health impact due to the large number of elderly at risk."

Subsequent studies have relied more on measures of skeletal lead than of blood lead because the half-life of lead in the latter is about 35 days while the half-life in the skeleton, depending on the assay location, can be as much as two decades. Skeletal measures, based on X-ray fluorescence (XRF) methods, have the virtue, in older adults, of providing a historical and cumulative index of exposure [9], a considerable advantage because lead is so embedded in the environment that epidemiological studies cannot readily distinguish the effects of recent from past exposures. In one study [10], which surveyed nearly 1,000 adults in Baltimore, Maryland, performance scores on a variety of neuropsychological tests were found to be associated with tibia lead concentrations (language, processing speed, eyehand coordination, executive functioning, verbal memory and learning, and visual memory). The mean blood lead level at the time of testing was $3.2 \mu \mathrm{g} / \mathrm{dL}$.

In another study of an elderly population in Boston [11], the investigators measured bone lead in about 1,100 men at various times between 1991 and 1999. In this population, performance over this period on a battery of neuropsychological tests worsened as bone lead increased. The most robust effects were reflected by performance and 


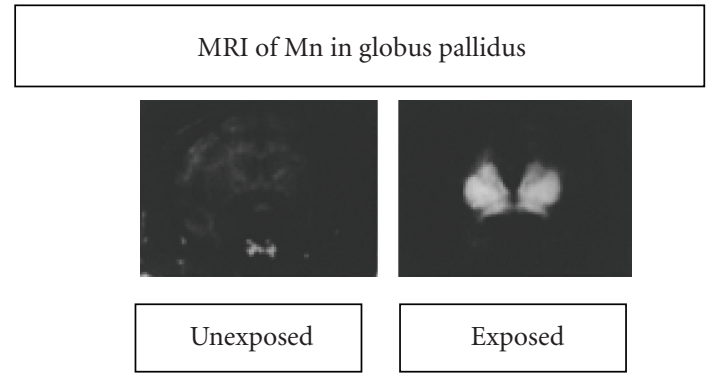

Figure 2: Magnetic resonance images of two monkeys, one exposed to inhaled manganese and one contol. They show that manganese achieves high concentrations in the global pallidus. Based on Newland and Weiss [12].

reaction time scores on visuospatial/visuomotor tests. Here, mean blood lead values lay in the range of $5 \mu \mathrm{g} / \mathrm{dL}$. The authors, in describing the rationale for their study and its implications for $\mathrm{AD}$, commented as follows: "Mild cognitive impairment is more prevalent than $\mathrm{AD}$ and is receiving increasing attention, not only as a possible intermediate stage on the path to $\mathrm{AD}$, but as an important deficit in its own right." The argument of Gilbert and Weiss [7] that acceptable lead levels be reduced applies to adults as well as children.

Because women had been underrepresented in lead studies, Weuve et al. [13] chose a population from the Nurses Health Study, which began in 1976. Over 90\% of the participants have continued to participate, completing mail questionnaires every two years. Nearly 600 women took part in the current study and ranged in age from 47 to 74 . They underwent cognitive testing and bone and blood lead assessment. Remarkably, mean blood lead values lay in the $2-3 \mu \mathrm{g} / \mathrm{dL}$ range. As noted by the authors, current values do not reflect the much higher exposures that prevailed in the past. Not until 1971 did the US Congress pass the Lead-Based Paint Poisoning Prevention Act, and not until 1986 was the phase out of lead from gasoline completed. As a result of this action, mean US blood-lead levels declined by 78 percent from 1978 to 1991. As noted earlier, however, the legacy of earlier environmental lead exposures remains implanted in the individual, either visibly in the skeleton, or epigenetically [14] or covertly, as silent neural damage that is not revealed until the compensatory capacity of the brain has been eroded by aging [15].

Generally, cognitive assessment occurred five years following the exposure assessments. The tests were conducted by trained nurses and administered by telephone interviews. The assessment battery consisted primarily of memory tests. As in the aging study in men, the concentration of lead in tibia bone was associated with worse overall cognitive performance. The authors translated this decrement into an age effect as follows: "Specifically, the average decrement in cognitive test scores we observed for each SD increase in tibia lead corresponded to the decrement in scores we observed for each 3-year increase in age among women in our study." Put another way, lead exposure induced premature aging, in essence shifting the score distribution to lower values.

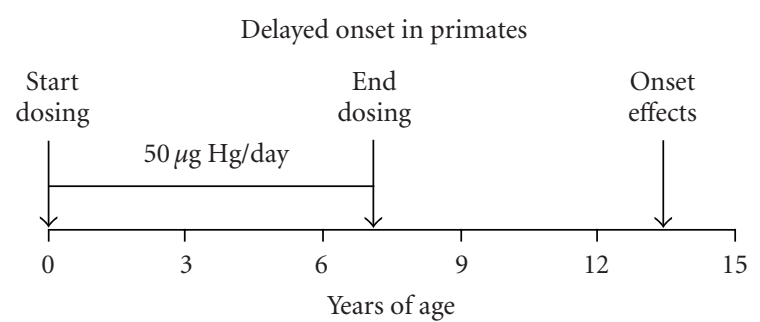

(a)

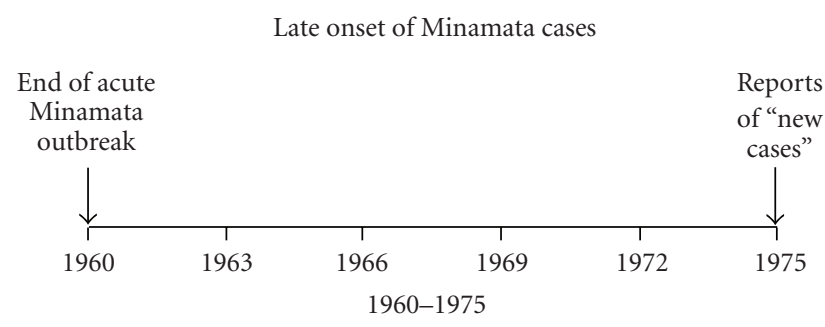

(b)

FIGURE 3: Depicting the phenomenon of delayed onset of neurotoxicity with methylmercury in nonnuman primates (a) and (b) in the Minamata population $[18,19]$.

Most neuropsychological tests are based on the needs of clinical practice and were designed to assist in the diagnosis in individual patients. Performance testing adopts a different point of view, one stemming from the needs of laboratory investigators, whose aim is to determine the effects of some experimental procedures on a specific behavior in a population sample. Proceeding from this standpoint, van Wijngaarden et al. [16] assessed a group of healthy subjects 55-67 years of age on a battery of behavioral tests derived from procedures originally developed in the animal laboratory. These procedures, part of the Cambridge Neuropsychological Test Automated Battery (CANTAB), were selected because they had been shown to reflect the kinds of impairment characteristic of $\mathrm{AD}$. They included delayed matching-to-sample (DMS), paired associate learning (PAL), and spatial recognition memory (SRM). In addition, the subjects completed the Montreal Cognitive Assessment (MoCA) as an index of MCI.

As in other recent studies, the authors relied on XRF of bone to capture an index of cumulative lead exposure. And, following conventional practice, they corrected for a number of covariates that might serve as confounders such as age, smoking, alcohol use, body mass index (BMI), and others. In this pilot sample of 47 subjects, the clearest associations with exposure were seen with the DMS procedure, which calls upon the subject to select, after a variable delay, one of four complex visual patterns that matches a single test pattern shown before the delay. A similar task revealed deficits in nonhuman primates exposed chronically to manganese [17]. The other two CANTAB tests (SRM and PAL) also showed significant associations with bone lead, but not as conspicuously as the DMS procedure. The MoCA inventory showed only a weak association. 


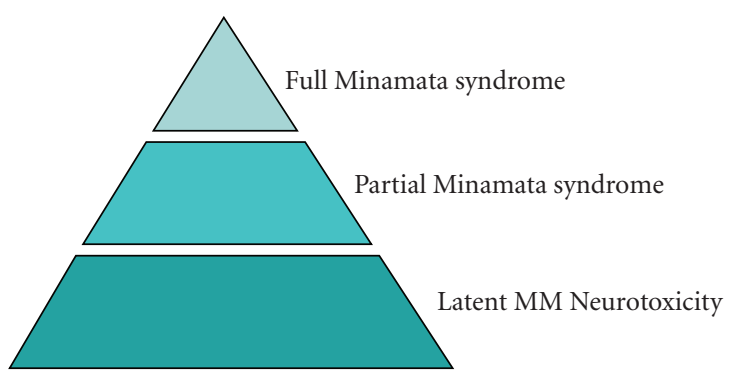

FIgURE 4: Schematic of scope of neurotoxic signs stemming from exposure to methylmercury. The full syndrome, as in Table 3, occurs with massive exposures. The primary current question, a population question, arises from the possible occurrence of subtle neuropsychological deficits that would only be evident with systematic testing; that is, latent effects.

The message conveyed by these recent publications tells us that historical and cumulative as well as current lead exposure must be considered a risk factor for neurobehavioral dysfunction, including a decline in cognitive performance. Translating these findings into the model depicted in Figure 1, it will be seen that lead exposure shifts the test score distribution toward lower values. The result is that a larger proportion of the population falls into the range of markedly diminished cognitive function - a basic criterion for dementia. Or, as noted earlier, because population indices of cognitive function also decline with age, it shifts the scores toward more advanced ages; that is, premature aging.

One other aspect of these data warrants comment, although we have no direct evidence of their impact in human populations. Environmental health specialists now view as a principle the notion that toxic exposures during early development create the foundation for adult disease. A substantial literature in neurotoxicology testifies to the validity of that principle for neurobehavioral function. Recent research links developmental lead exposure to elevated deposits of $\beta$-amyloid deposits in both aged rats [20] and monkeys [21], leading the authors to propose a twostage model for the genesis of dementia. Stage one is triggered by a condition such as lead exposure, which inscribes an epigenetic footprint in the form of altered patterns of DNA methylation. Stage two occurs late in life, possibly a result of further stress, including diminished brain compensatory capacity. Acting in concert, this combination of stressors is postulated to evoke the pathogenic processes that underlie dementia. Whether and how such a sequence eventually results in impaired performance on behavioral tests late in life is a question that warrants further exploration.

\section{Manganese}

Manganese $(\mathrm{Mn})$ has been recognized as a neurotoxic metal for over 150 years. Exposure to high levels of inhaled manganese, as in miners working in an environment laden with dust from manganese ore $\left(\mathrm{MnO}_{2}\right)$, leads to a constellation of psychological and motor symptoms depicted in Table 2. The psychological changes are known as "locura
TABLE 3: Scope of methylmercury poisoning experienced in Japan beginning in the 1950s. This syndrome became known as Minamata Disease. Later, when the effects of prenatal exposure became evident, a new category, Fetal Minamata Disease, was established.

\begin{tabular}{l}
\hline SENSORY \\
\hline (i) Paresthesia (numbness and tingling) \\
(ii) Pain in limbs \\
(iii) Visual disturbances (field constriction) \\
(iv) Hearing disturbances \\
(v) Asterognosis (discrimination by touch) \\
\hline MOTOR \\
(i) Disturbances of gait \\
(ii) Weakness, leg unsteadiness, falling \\
(iii) Thick, slurred speed (dysarthria) \\
(iv) Tremor \\
OTHER \\
Headaches, rashes, "mental disturbance"
\end{tabular}

manganica" in the South American manganese mines where manganism is endemic. Many of the motor and postural signs are consistent with those of Parkinson's disease (PD), an overlap that has led to debates about whether manganese neurotoxicity is truly a form of PD. Most observers agree that the movement disorders induced by manganese exposure and idiopathic Parkinsonism differ in fundamental respects. PD patients typically walk with shuffling gait. In manganism, patients may walk with an almost theatrical high-stepping gait. Dopamine replacement can attenuate PD signs; it has little effect on manganism. In PD, degeneration is seen primarily in the substantia nigra; in manganism, it is seen primarily in the globus pallidus.

Because manganese is paramagnetic, it can be localized in the brain by magnetic resonance imaging (MRI). Figure 2 shows two images, one from a monkey exposed by inhalation and one from a control subject [12]. The figure shows the tendency of inhaled manganese to collect in the globus pallidus. A similar pattern of distribution occurs in humans as well [22].

The overtly observable manifestations of overt manganese poisoning have obscured the more subtle, more widespread consequences of low-level exposures, much as the overt signs of Parkinson's disease have tended to eclipse its neuropsychological accompaniments such as cognitive impairment and depression. In parallel, at lower levels of exposure than those associated with mining, such as those experienced by welders exposed to manganese fumes from welding rods, or to fumes by workers in ferromanganeseprocessing plants, or in areas where air or water levels are elevated, the manifestations are far less dramatic. They do not rise to the level of clinical detection. Instead, they require the application of neuropsychological tests.

Two aspects of manganese neurotoxicity render it a more difficult question to resolve than is the case with lead and mercury. First, it is an essential element. It is required to maintain health as well as to support growth and development. It is present in our tissues and is necessary for 
certain biochemical reactions. Environmental levels cannot be reduced to zero. Second, perhaps because it is an essential element, no unambiguous biological marker, of the kind we have for lead and mercury, is available. Blood Mn seems to be the best commonly available index of exposure.

From the standpoint of general population risk, the main questions posed by manganese exposure lie more in the realm of neuropsychological function than in the realm of motor function. It must be recognized, however, that the two realms are intertwined. Both spatially and functionally, the brain is highly interactive. Simply because the globus pallidus is part of the basal ganglia does not isolate it from cognitive pathways. For example, McNab and Klingberg [23] demonstrated, with fMRI, how working memory depends on pallidal and frontal cortical cooperation, so to speak.

Inhalation is the predominant mode of exposure leading to neurotoxicity. For chronically exposed individuals, the latency to clinical manganism is highly variable, dependent on ambient concentrations, particle size, and individual susceptibility. In some instances, it apparently surfaces long after exposure has ceased. Because incipient poisoning may be expressed primarily by subjective symptoms such as mood changes, a connection to manganese is easily overlooked. This is the rationale for neuropsychological testing. As in other neurotoxic syndromes, systematic testing is more sensitive than the typical neurological examination. It is why the cognitive effects of manganese neurotoxicity were virtually ignored until the last two decades. They were submerged by the more blatant signs such as the motor abnormalties and the striking emotional displays such as hysterical laughing and weeping. More recently, however, we have begun to realize that cognitive deficits remain and may even worsen, years after exposure has ceased [24]. Perhaps even more worrisome, cognitive and associated measures of neurobehavioral function have been documented in children [25]. Such losses, we now recognize, may persist for a lifetime, perhaps lying dormant until advanced age.

The cognitive deficiencies embrace a variety of measures. They include diminished attention, reduced scores on tests of working memory, lower scores on intelligence tests, impaired learning, and slowed response speed. The recent literature expands these findings, as exemplified by two recent papers.

Diminished neuropsychological function (such as attention and memory) is detectable at about the same exposure levels as the motor deficits typically thought of as most characteristic of manganism. Bowler et al. [24] examined 47 welders and 42 controls on a variety of tests: the Wechsler Adult Intelligence Scale (WAIS-III), the Wechsler Memory Scale (WMS-III), the Boston Naming Test, the Wide Range Achievement Test (WRAT-3), Cancellation H, Trail Making Tests A and B, Auditory Consonant Trigrams, Stroop (color naming) test, Rey-Osterreith, Animal Naming, Controlled Oral Word Association (COWAT), Test of Memory Malingering, Rey 15-item, Fingertapping, Grooved Pegboard, Dynamometer, Visual Attention Test, and the Lanthony d-15 Color Vision test. They saw diminished performance, among the welders, in motor skills, visuomotor tracking speed and information processing, working memory, verbal skills (COWAT), delayed memory, and visuospatial skills.
Concentrations of manganese in the globus pallidus are correlated with neuropsychological test performance. Chang et al. [22] administered a battery of such tests to subjects who had also been evaluated by MRI. To 43 asymptomatic male welders and 29 age- and sex-matched healthy controls, they administered the following tests: Digit symbol, auditory verbal learning test (delayed recall), complex figure test (copy and immediate recall), digit span, verbal fluency test, Stroop test, grooved pegboard, finger tapping, and frequency dispersion and harmonic index of tremor. They also assessed olfactory function and mood. Welders attained lower scores than controls on several cognitive tests, but, most interesting, multiple regression analyses revealed pallidal index to be a better predictor of performance in welders than blood Mn. (Pallidal index was defined as the ratio of the signal intensity of the globus pallidus to that of the subcortical frontal white matter in axial T1-weighted MRI planes.).

Nonhuman primates are viewed as the most appropriate animal model for studies of manganese neurotoxicity because of their similarities to humans in brain anatomy and neurobehavioral function. Two recent publications indicate the depth and extent of understanding that can be achieved by making use of these characteristics.

Schneider et al. [17] trained Cynomologous monkeys on three memory tasks: spatial working memory (delayed position choice), nonspatial working memory (delayed matchto-sample), and reference memory (pattern discrimination). Once trained, they were placed on a regimen of intravenous manganese sulfate injections over a period of about 230 days. When tested at the end of this period, the treated animals displayed mild deficits in spatial memory, greater deficits in nonspatial memory, and no deficits in reference memory. By analyzing regional Mn concentrations, the experimenters determined a significant inverse relationship between working memory task performance and Mn levels.

In a direct quest for associations between manganese exposure and Alzheimer's disease, Guilarte [26] conducted a gene array analysis of frontal cortex tissue from the same animals. Of the 61 upregulated genes, the most highly elevated was Amyloid- $\beta$ Precursor-like Protein 1 (APLP1), a member of the Amyloid Precursor Protein (APP) family. Along with this finding, immunochemistry showed the presence of Amyloid- $\beta$ plaques, an unexpected finding given that the subjects were only $6-8$ years of age. In addition, immunochemistry revealed the presence of $\alpha$-synuclein aggregates, which have been linked to $\mathrm{PD}$ as well as AD. These findings, then, link the Mn-induced $\beta$-amyloid deposits to impaired memory function in a species generally considered to be an especially useful model for extrapolation to humans.

Because manganese exposure seems primarily to occur via inhalation, I would be remiss in not observing current data about the mechanics of ambient particulates. They have received considerable attention as risk factors for cardiovascular disease. We are now aware that the smallest particles, termed ultrafine or nanoparticles, may directly enter the central nervous system as they travel along the olfactory nerve. In rats exposed to manganese oxide ultrafine particles for 12 days, Elder et al. [27] found these particles 


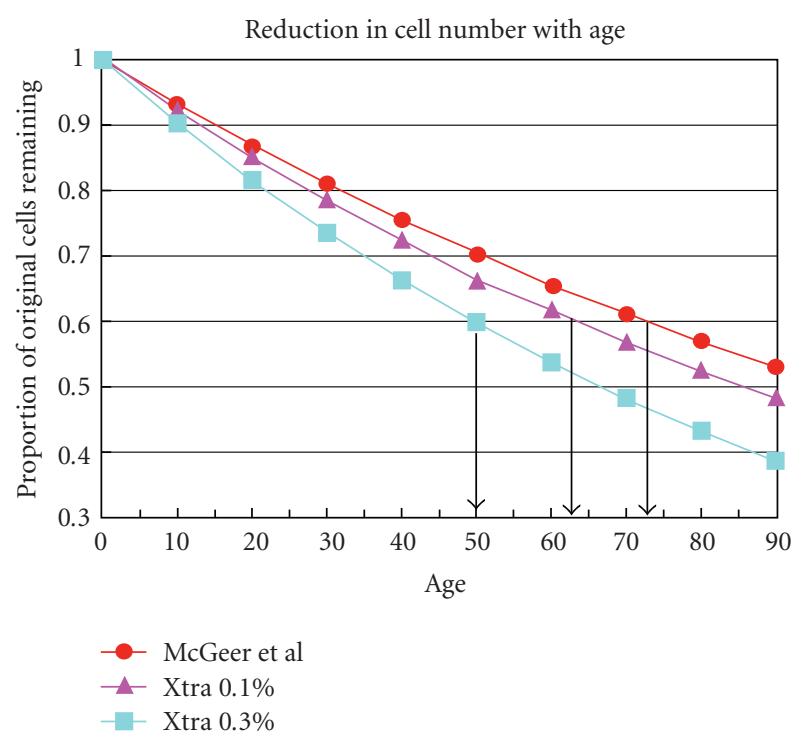

FIGURE 5: Substantia nigra cell populations diminish with age, as shown by McGeer et al. [31]. At about age 72, about $40 \%$ of the cells have been lost. If the decline is accelerated by $0.1 \%$ per year, such a loss would occur at about age 64 .

translocating into the striatum, frontal cortex, and cerebellum. This is an exposure pathway that needs considerably more attention from the standpoint of neurodegenerative disease $[28,29]$.

\section{Methylmercury}

An ecological disaster in Japan provided one of the earliest signals about how chemical contamination of the environment could engender major threats to human health, particularly brain function. Perhaps even more than Rachel Carson's Silent Spring, which lamented the disappearance of bird song [30], it framed its message in images of deformed children and crippled adults. The chemical contaminant was methylmercury and the site was in Japan, on the island of Kyushu, in the small fishing village of Minamata. There, beginning in the 1950s, the inhabitants began to suffer from a strange neurological disease traced to methylmercury contamination of fish and shellfish from Minamata bay. The first cases were sporadic, but, with time, more and more cases became evident and finally led to an investigation that indicted mercury dumped into the bay by a chemical factory. The clinical signs of methylmercury poisoning are listed in Table 3.

The story has been told many times, but none as graphically as in the photographic essay by E. Smith and A. Smith [32]. Since then, methylmercury has been the subject of several thousand scientific papers and still presents numerous mysteries [33].

The overwhelming proportion of publications dealing with methylmercury neurotoxicity is devoted to early brain development $[34,35]$. Although one of the earliest contributions to methylmercury research reported that perinatal

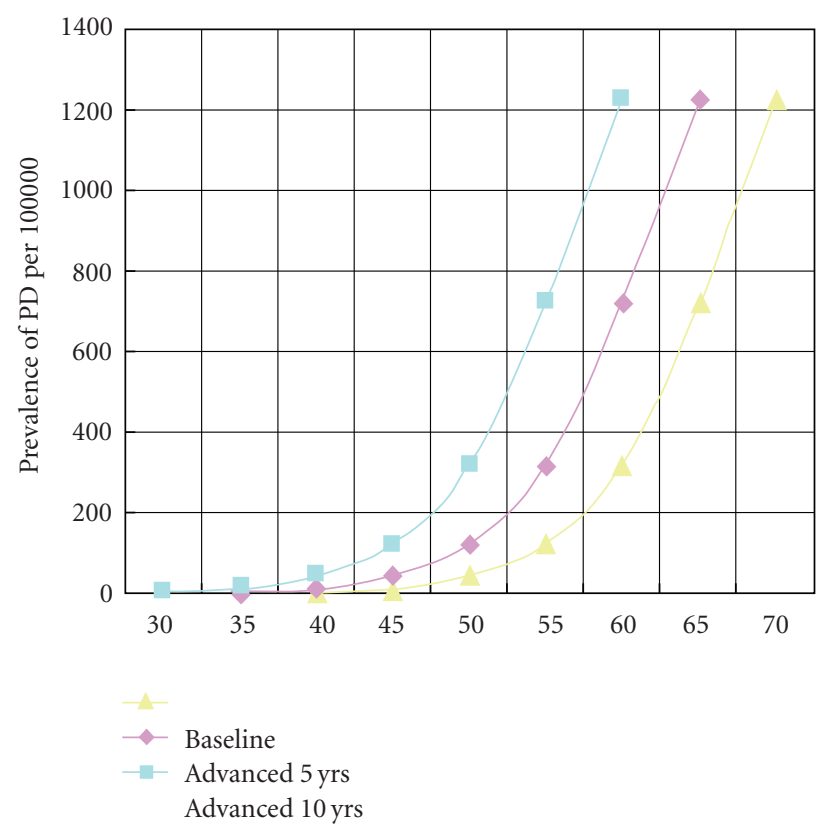

Figure 6: The chart demonstrates how the age-adjusted functions of a disorder such as Parkinson's disease are modified by an acceleration of cell loss as depicted in Figure 5. At age 65 years, the prevalence without acceleration is, based on available data, about $700 / 100,000$. If advanced by 5 years, the prevalence would be $1,200 / 100,000$.

exposure in mice could induce adverse consequences that might erupt eventually in advanced age [36], that portion of the lifespan has still received relatively little attention.

The paucity of this literature fails to match the scope of the problem. We are exposed to methylmercury almost exclusively through fish consumption. One exception was the mass poisoning in Iraq in 1971-1972, but its source was grain treated with a methylmercury fungicide $[37,38]$. Exposures therefore tend to continue through a lifetime in those whose diet includes fish. Even as a consequence of developmental exposure alone, we must address the possibility that exposures early in life may produce undetected, latent, or "silent" damage that emerges only when the functional capacities of the nervous system are challenged by other conditions, such as aging $[39,40]$ as was noted earlier for the two-stage (or two-hit) model for lead. Figure 3 shows two findings bearing on this question. First, that for many years after the first cases were documented, new cases continued to appear, possibly because of the combination of aging and silent damage. Second, in nonhuman primates exposed during early development to lead [18] and methylmercury [19], a long period elapsed before neurotoxic effects made an overt appearance.

The most serious gap is in our understanding of methylmercury's possible effects on cognitive function during aging lies in the domain of neuropsychological assessment. Perhaps the absence of such data can be ascribed to the most conspicuous signs of poisoning in adults; that is, those listed in Table 3. They are so flagrant that more subtle outcomes, such as cognitive dysfunction, were 
not pursued. No neuropsychologists were enlisted in the numerous studies conducted by Japanese physicians and scientists in the years following the onset of the poisoning. Surveys of elderly Japanese in areas marked by elevated exposures, as determined by biomarkers such as hair, or less precisely, by geographic location, have tended to focus on endpoints, such as paresthesias and ataxia, that are part of a conventional neurological examination [41, 42]. In two studies that evaluated subjective complaints, however, "forgetfulness," a cognitive term, emerged as a common complaint $[43,44]$.

Once systematic neuropsychological assessments were undertaken, however, they uncovered evidence of impairment. Carta et al. [45] compared a group of 22 adult male tuna fish eaters with 22 controls, both about 50 years of age, on a neurobehavioral test battery. The tuna eaters exhibited much higher levels of organic mercury in blood and hair. Two cognitive tests differentiated the two groups statistically, digit-symbol and color word reaction time, while overall performance was inversely correlated with biomarker levels.

Chang et al. [46] took advantage of a Taiwanese population living in the vicinity of an abandoned chlor-alkali factory; such factories typically use big tanks of elemental mercury as an electrode. Fish in the area were heavily contaminated because of previous mercury waste discharges into the adjacent river where, as in most waterways, microorganisms in bottom sediment convert the inorganic mercury into the methyl species. Measures of total and organic mercury in blood indicated somewhat elevated levels compared to US residents, but close to those of residents of the Faroe Islands and the Republic of Seychelles, both sites of large prospective studies of methylmercury effects on brain development $[34,35]$. For the neurobehavioral assessments, the authors relied upon the Cognitive Abilities Screening Instrument (CASI) and the Mini-Mental State Exam (MMSE). They then divided the population in two: high blood and low blood methylmercury values. The two groups achieved significantly different scores on the CASI and MMSE, with the low ones superior. Although all the subtests showed better scores by those with low values, three subtests yielded significant differences on their own: remote memory, recent memory, and mental manipulation.

The published literature contains other studies pointing in the same direction. For example, Yokoo et al. [47], studying an Amazonian population, found correlations between hair concentrations of methylmercury with performance on several neuropsychological tests. It seems, then, even with this small group of studies, that adult brains are not as resistant to methylmercury neurotoxicity, compared to developing brains, as had been believed. Chronic exposure, combined with the diminished performance associated with aging, may account for this unexpected sensitivity.

All three studies present the same conundrum. As with all fish-consuming populations, unless they are followed from conception onwards, it becomes virtually impossible to differentiate the effects of current exposure from those occurring early in development.

The laboratory animal literature supports the view that advanced age is a period during which the damage inflicted by developmental or chronic exposure may first emerge. Spyker [36] showed a variety of adverse effects such as obesity, kyphosis, and impaired locomotion in old mice exposed prenatally. Newland et al. [48, 49] found that that the adult consequences of developmental exposure in rats can be reflected in how precisely subjects adjust to new behavioral demands. In nonhuman primates, Rice [19] and Burbacher et al. [50] found enduring effects of developmental exposure on coordination and sensory function in monkeys that appeared only many years after cessation of dosing.

The Minamata progression, from its first focus on clinical poisoning to determining who was actually a victim to the more recent question of subtle neuropsychological effects, can be depicted by the pyramid in Figure 4, which actually represents the kind of questions evoked by environmental contaminants in general. It is often the case that only after we see obvious signs of adverse effects do we begin to probe further and to search for the predecessors of such effects.

\section{Discussion}

In 1956, in the midst of the continuing controversy over the Minamata disaster, the Japanese government asked Kumamoto University, located nearby, to undertake studies of the outbreak. The researchers affirmed the connection between the outbreak of the disease and methylmercury contamination resulting from waste discharges into Minamata Bay by the Chisso chemical plant. The question of compensation for victims then arose: who was injured and what was the evidence? In 1973, the Research Committee on Minamata Disease of Kumamoto University described its dilemma in assigning compensation in these terms as follows:
"...the problem about the relationship between a small amount of methylmercury pollution for a long period and its accumulation in the brain still remains obscure...Subclinical Minamata disease was sometimes revealed by detectable symptoms during the aging process...The sub- clinical Minamata disease...could be called a delayed type of Minamata disease in aged peo- ple."

This statement highlights the issues posed in this paper. Neurodegenerative disorders such as Alzheimer's disease are disorders of aging, and the primary risk factor, except for the familial form, is age. To what extent their etiology and, perhaps more narrowly, their rate of progression or latency to clinical emergence is fostered by environmental contaminants is all but unknown at present. We can, however, note how aging intersects with neurotoxicant exposure [51]. Many manifestations of neurotoxicity, such as impaired sensory acuity, mimic the natural course of aging. Some adverse effects may arise for the first time in advanced age because it is a period of declining compensatory margins. With aging, for example, synaptic density decreases $[52,53]$. Attrition of nerve cells also occurs naturally as the brain ages but is also 
accompanied by dendritic sprouting in those that remain. Damage incurred earlier in life, as in mild polio infections that seemed to fade, may emerge late in life in the form of the postpolio syndrome. Could parallel processes be induced by neurotoxicants such as methylmercury?

(i) Early development, it now seems clear, is not the only life stage during which the brain exhibits intensified responses to the adverse effects of chemicals. Vulnerability to toxic processes rises again late in life; recovery from damage is sluggish, and pharmacokinetic and metabolic variables change with aging in ways that recapitulate the imperfect defenses deployed by the immature organism.

(ii) "Aging" is not a mechanistic explanation. Events occurring during life must account for the changes. Older brains are already high-maintenance properties, so that exposure to substances with neurotoxic properties, such as certain metals, may accelerate the process or exploit its dwindling capacities to resist their effects. From this vantage point, toxicants can act in three ways to depress function during advanced age; they may interfere with brain development, leaving a legacy of diminished redundancy not apparent until it is further compromised during aging; they may hasten the progressive erosion of function observed with certain abilities; they may exert greater effects in the aging brain because the aging nervous system has already undergone a reduction in its ability to withstand toxic challenges.

These arguments can be illustrated by Figures 5 and 6 . Figure 5 is based on data from McGeer et al. [31], which trace the decline in substantia nigra cell number that occurs with aging. Using the same rationale as that of Weiss and Simon [39], it compares the McGeer data with two models of rate of decline. One shows an additional acceleration of $1 \%$ per year, the other one of $3 \%$ per year. At the $1 \%$ rate, remaining cell number shows a fall of $40 \%$ at about age 64 while the baseline rate of decline would not reach that level until age 72 .

Figure 6 converts these speculative projections into their public health implications. It plots the age-indexed prevalence of Parkinson's disease for a baseline population against one that has been advanced by 5 years and another that has been advanced by 10 years. At age 65 , the prevalence comes to about 700/100,000 for the baseline population. For the population advanced by 5 years, the figure becomes about $1,200 / 100,000$. This phenomenon is what happens with disorders whose prevalence increases with age [54].

Figures 5 and 6 convey the core message of this paper. It misleads us to conceive of environmental contaminants such as metals as causes of neurodegenerative diseases such as $\mathrm{AD}$. To truly establish such a classification requires data from several biological levels ranging from epidemiology to molecular mechanisms. With the three metals surveyed here, however, the available information is sturdy enough that we can label them as potential contributors or risk factors for the onset or progression of the disorder. Given the paucity of information we now possess about the etiology of neurodegenerative disorders, they warrant further investigation about their influence on aging.

\section{Acknowledgment}

Preparation supported in part by research Grant no. RC2 ES018736 and Center Grant no. ES01247 from the National Institute of Environmental Health Sciences.

\section{References}

[1] D. K. Lahiri, N. H. Zawia, N. H. Greig, K. Sambamurti, and B. Maloney, "Early-life events may trigger biochemical pathways for Alzheimer's disease: the "LEARn" model," Biogerontology, vol. 9, no. 6, pp. 375-379, 2008.

[2] R. Brookmeyer, S. Gray, and C. Kawas, "Projections of Alzheimer's disease in the United States and the public health impact of delaying disease onset," American Journal of Public Health, vol. 88, no. 9, pp. 1337-1342, 1998.

[3] B. Weiss, "Neurobehavioral toxicity as a basis for risk assessment," Trends in Pharmacological Sciences, vol. 9, no. 2, pp. 59-62, 1988.

[4] T. Hedden and J. D. E. Gabrieli, "Insights into the ageing mind: a view from cognitive neuroscience," Nature Reviews Neuroscience, vol. 5, no. 2, pp. 87-96, 2004.

[5] L. L. Drag and L. A. Bieliauskas, "Contemporary review 2009: cognitive aging," Journal of Geriatric Psychiatry and Neurology, vol. 23, no. 2, pp. 75-93, 2010.

[6] L. E. Hebert, P. A. Scherr, J. L. Bienias, D. A. Bennett, and D. A. Evans, "Alzheimer disease in the US population: prevalence estimates using the 2000 census," Archives of Neurology, vol. 60, no. 8, pp. 1119-1122, 2003.

[7] S. G. Gilbert and B. Weiss, "A rationale for lowering the blood lead action level from 10 to $2 \mu \mathrm{g} / \mathrm{dL}$," NeuroToxicology, vol. 27, no. 5, pp. 693-701, 2006.

[8] S. B. Muldoon, J. A. Cauley, L. H. Kuller et al., "Effects of blood lead levels on cognitive function of older women," Neuroepidemiology, vol. 15, no. 2, pp. 62-72, 1996.

[9] H. Hu, R. Shih, S. Rothenberg, and B. S. Schwartz, "The epidemiology of lead toxicity in adults: measuring dose and consideration of other methodologic issues," Environmental Health Perspectives, vol. 115, no. 3, pp. 455-462, 2007.

[10] R. A. Shih, T. A. Glass, K. Bandeen-Roche et al., "Environmental lead exposure and cognitive function in communitydwelling older adults," Neurology, vol. 67, no. 9, pp. 1556$1562,2006$.

[11] M. G. Weisskopf, S. P. Proctor, R. O. Wright et al., "Cumulative lead exposure and cognitive performance among elderly men," Epidemiology, vol. 18, no. 1, pp. 59-66, 2007.

[12] M. C. Newland and B. Weiss, "Persistent effects of manganese on effortful responding and their relationship to manganese accumulation in the primate globus pallidus," Toxicology and Applied Pharmacology, vol. 113, no. 1, pp. 87-97, 1992.

[13] J. Weuve, S. A. Korrick, M. A. Weisskopf et al., "Cumulative exposure to lead in relation to cognitive function in older women," Environmental Health Perspectives, vol. 117, no. 4, pp. 574-580, 2009.

[14] D. K. Lahiri, B. Maloney, and N. H. Zawia, "The LEARn model: an epigenetic explanation for idiopathic neurobiological diseases," Molecular Psychiatry, vol. 14, no. 11, pp. 9921003, 2009. 
[15] B. Weiss, T. W. Clarkson, and W. Simon, "Silent latency periods in methylmercury poisoning and in neurodegenerative disease," Environmental Health Perspectives, vol. 110, supplement 5, pp. 851-854, 2002.

[16] E. van Wijngaarden, J. R. Campbell, and D. A. Cory-Slechta, "Bone lead levels are associated with measures of memory impairment in older adults," NeuroToxicology, vol. 30, no. 4, pp. 572-580, 2009.

[17] J. S. Schneider, E. Decamp, K. Clark, C. Bouquio, T. Syversen, and T. R. Guilarte, "Effects of chronic manganese exposure on working memory in non-human primates," Brain research, vol. 1258, pp. 86-95, 2009.

[18] D. C. Rice and K. F. Karpinski, "Lifetime low-level lead exposure produces deficits in delayed alternation in adult monkeys," Neurotoxicology and Teratology, vol. 10, no. 3, pp. 207-214, 1988.

[19] D. C. Rice, "Evidence for delayed neurotoxicity produced by methylmercury," NeuroToxicology, vol. 17, no. 3-4, pp. 583596, 1996.

[20] MD. R. Basha, M. Murali, H. K. Siddiqi et al., "Lead $(\mathrm{Pb})$ exposure and its effect on APP proteolysis and $\mathrm{A} \beta$ aggregation," FASEB Journal, vol. 19, no. 14, pp. 2083-2084, 2005.

[21] J. Wu, MD. R. Basha, B. Brock et al., "Alzheimer's Disease (AD)-like pathology in aged monkeys after infantile exposure to environmental metal lead $(\mathrm{Pb})$ : evidence for a developmental origin and environmental link for AD," Journal of Neuroscience, vol. 28, no. 1, pp. 3-9, 2008.

[22] Y. Chang, Y. Kim, S. T. Woo et al., "High signal intensity on magnetic resonance imaging is a better predictor of neurobehavioral performances than blood manganese in asymptomatic welders," NeuroToxicology, vol. 30, no. 4, pp. 555-563, 2009.

[23] F. McNab and T. Klingberg, "Prefrontal cortex and basal ganglia control access to working memory," Nature Neuroscience, vol. 11, no. 1, pp. 103-107, 2008.

[24] R. M. Bowler, S. Gysens, E. Diamond, S. Nakagawa, M. Drezgic, and H. A. Roels, "Manganese exposure: neuropsychological and neurological symptoms and effects in welders," NeuroToxicology, vol. 27, no. 3, pp. 315-326, 2006.

[25] G. A. Wasserman, X. Liu, F. Parvez et al., "Water manganese exposure and children's intellectual function in Araihazar, Bangladesh," Environmental Health Perspectives, vol. 114, no. 1, pp. 124-129, 2006.

[26] T. R. Guilarte, “APLP1, Alzheimer's-like pathology and neurodegeneration in the frontal cortex of manganese-exposed non-human primates," NeuroToxicology, vol. 31, no. 5, pp. 572-574, 2010.

[27] A. Elder, R. Gelein, V. Silva et al., "Translocation of inhaled ultrafine manganese oxide particles to the central nervous system," Environmental Health Perspectives, vol. 114, no. 8, pp. 1172-1178, 2006.

[28] M. L. Block and L. Calderón-Garcidueñas, "Air pollution: mechanisms of neuroinflammation and CNS disease," Trends in Neurosciences, vol. 32, no. 9, pp. 506-516, 2009.

[29] L. Calderón-Garcidueñas, A. C. Solt, C. Henríquez-Roldán et al., "Long-term air pollution exposure is associated with neuroinflammation, an altered innate immune response, disruption of the blood-brain barrier, ultrafine particulate deposition, and accumulation of amyloid $\beta-42$ and $\alpha$-synuclein in children and young adults," Toxicologic Pathology, vol. 36, no. 2, pp. 289-310, 2008.

[30] R. Carson, Silent Spring, Houghton Mifflin, New York, NY, USA, 1962.
[31] P. L. McGeer, S. Itagaki, H. Akiyama, and E. G. McGeer, "Rate of cell death in parkinsonism indicates active neuropathological process," Annals of Neurology, vol. 24, no. 4, pp. 574-576, 1988.

[32] E. Smith and A. Smith, Minamata, Apeture (Grossman), New York, NY, USA, 1975.

[33] B. Weiss, "Why methylmercury remains a conundrum 50 years after Minamata," Toxicological Sciences, vol. 97, no. 2, pp. 223225, 2007.

[34] P. W. Davidson, G. J. Myers, C. Cox et al., "Methylmercury and neurodevelopment: longitudinal analysis of the Seychelles child development cohort," Neurotoxicology and Teratology, vol. 28, no. 5, pp. 529-535, 2006.

[35] F. Debes, E. Budtz-Jørgensen, P. Weihe, R. F. White, and P. Grandjean, "Impact of prenatal methylmercury exposure on neurobehavioral function at age 14 years," Neurotoxicology and Teratology, vol. 28, no. 3, pp. 363-375, 2006.

[36] J. Spyker, "Behavioral teratology and toxicology," in Behavioral Toxicology, B. Weiss and V. G. Laties, Eds., pp. 311-349, Plenum, New York, NY, USA, 1975.

[37] F. Bakir, S. F. Damluji, and I. Amin Zaki, "Methylmercury poisoning in Iraq: an interuniversity report," Science, vol. 181, no. 4096, pp. 230-241, 1973.

[38] T. W. Clarkson, L. Amin Zaki, and S. K. Al Tikriti, "An outbreak of methylmercury poisoning due to consumption of contaminated grain," Federation Proceedings, vol. 35, no. 12, pp. 2395-2399, 1976.

[39] B. Weiss and W. Simon, "Quantitative perspectives on the long-term toxicity of methylmercury and similar poisons," in Behavioral Toxicology, B. Weiss and V. G. Laties, Eds., pp. 429435, Plenum, New York, NY, USA, 1975.

[40] B. Weiss, T. W. Clarkson, and W. Simon, "Silent latency periods in methylmercury poisoning and in neurodegenerative disease," Environmental Health Perspectives, vol. 110, no. 5, pp. 851-854, 2002.

[41] S. Ekino, M. Susa, T. Ninomiya, K. Imamura, and T. Kitamura, "Minamata disease revisited: an update on the acute and chronic manifestations of methyl mercury poisoning," Journal of the Neurological Sciences, vol. 262, no. 1-2, pp. 131-144, 2007.

[42] T. Yorifuji, T. Tsuda, S. Takao, and M. Harada, "Long-term exposure to methylmercury and neurologic signs in Minamata and neighboring communities," Epidemiology, vol. 19, no. 1, pp. 3-9, 2008.

[43] Y. Kinjo, H. Higashi, A. Nakano, M. Sakamoto, and R. Sakai, "Profile of subjective complaints and activities of daily living among current patients with Minamata disease after 3 decades," Environmental Research, vol. 63, no. 2, pp. 241-251, 1993.

[44] Y. Fukuda, K. Ushijima, T. Kitano, M. Sakamoto, and M. Futatsuka, "An analysis of subjective complaints in a population living in a methylmercury-polluted area," Environmental Research, vol. 81, no. 2, pp. 100-107, 1999.

[45] P. Carta, C. Flore, R. Alinovi et al., "Sub-clinical neurobehavioral abnormalities associated with low level of mercury exposure through fish consumption," NeuroToxicology, vol. 24, no. 4-5, pp. 617-623, 2003.

[46] J. W. Chang, M. C. Pai, H. L. Chen, H. R. Guo, H. J. Su, and C. C. Lee, "Cognitive function and blood methylmercury in adults living near a deserted chloralkali factory," Environmental Research, vol. 108, no. 3, pp. 334-339, 2008.

[47] E. M. Yokoo, J. G. Valente, L. Grattan, S. L. Schmidt, I. Platt, and E. K. Silbergeld, "Low level methylmercury exposure 
affects neuropsychological function in adults," Environmental Health, vol. 2, no. 1, pp. 1-11, 2003.

[48] M. C. Newland, P. A. Reile, and J. L. Langston, "Gestational exposure to methylmercury retards choice in transition in aging rats," Neurotoxicology and Teratology, vol. 26, no. 2, pp. 179-194, 2004.

[49] M. C. Newland, E. M. Paletz, and M. N. Reed, "Methylmercury and nutrition: adult effects of fetal exposure in experimental models," NeuroToxicology, vol. 29, no. 5, pp. 783-801, 2008.

[50] T. M. Burbacher, K. S. Grant, D. B. Mayfield, S. G. Gilbert, and D. C. Rice, "Prenatal methylmercury exposure affects spatial vision in adult monkeys," Toxicology and Applied Pharmacology, vol. 208, no. 1, pp. 21-28, 2005.

[51] B. Weiss, "Vulnerability to pesticide neurotoxicity is a lifetime issue," NeuroToxicology, vol. 21, no. 1-2, pp. 67-74, 2000.

[52] H. Duan, S. L. Wearne, A. B. Rocher, A. Macedo, J. H. Morrison, and P. R. Hof, "Age-related dendritic and spine changes in corticocortically projecting neurons in macaque monkeys," Cerebral Cortex, vol. 13, no. 9, pp. 950-961, 2003.

[53] P. R. Hof and J. H. Morrison, "The aging brain: morphomolecular senescence of cortical circuits," Trends in Neurosciences, vol. 27, no. 10, pp. 607-613, 2004.

[54] B. Weiss, "Economic implications of manganese neurotoxicity," NeuroToxicology, vol. 27, no. 3, pp. 362-368, 2006. 


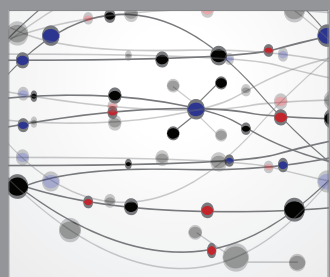

The Scientific World Journal
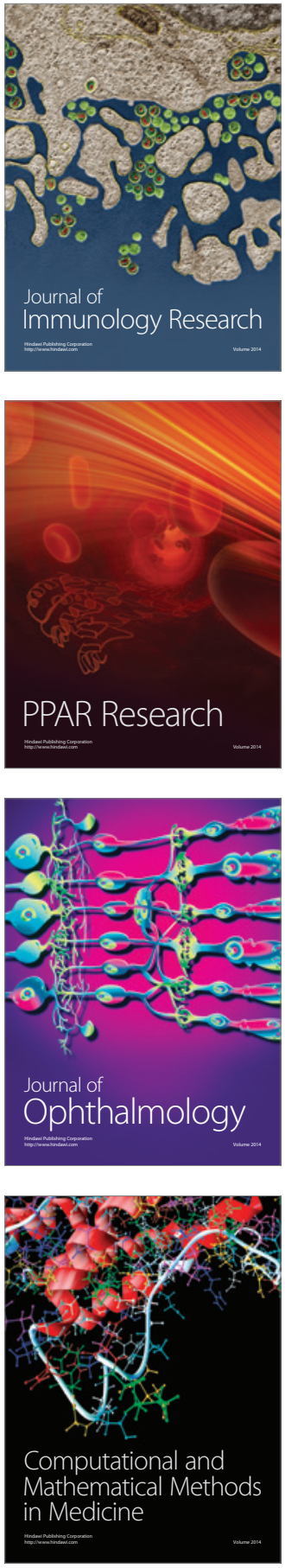

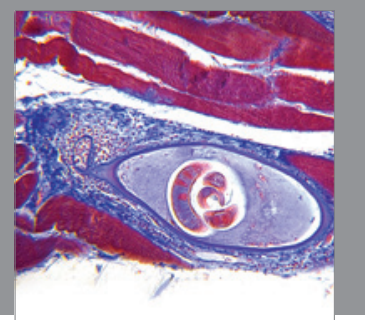

Gastroenterology

Research and Practice
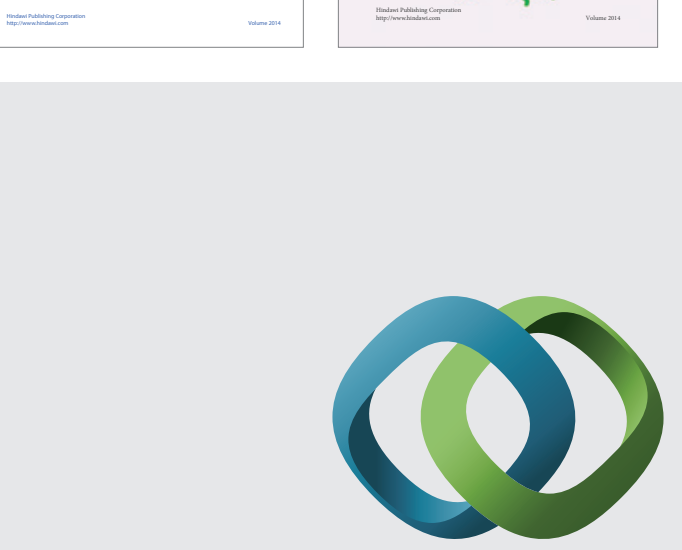

\section{Hindawi}

Submit your manuscripts at

http://www.hindawi.com
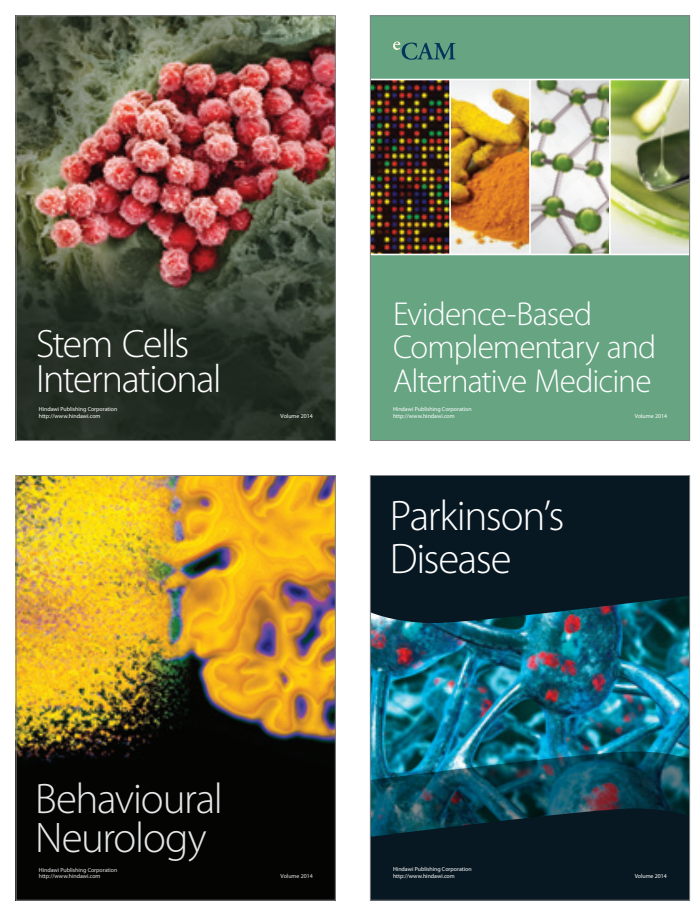

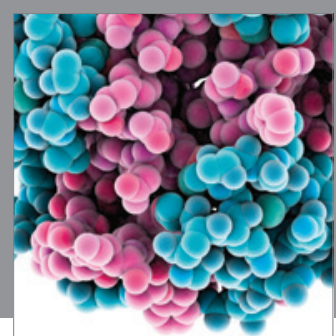

Journal of
Diabetes Research

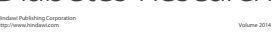

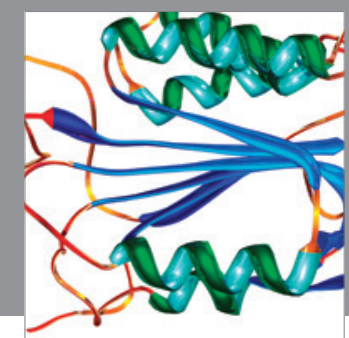

Disease Markers
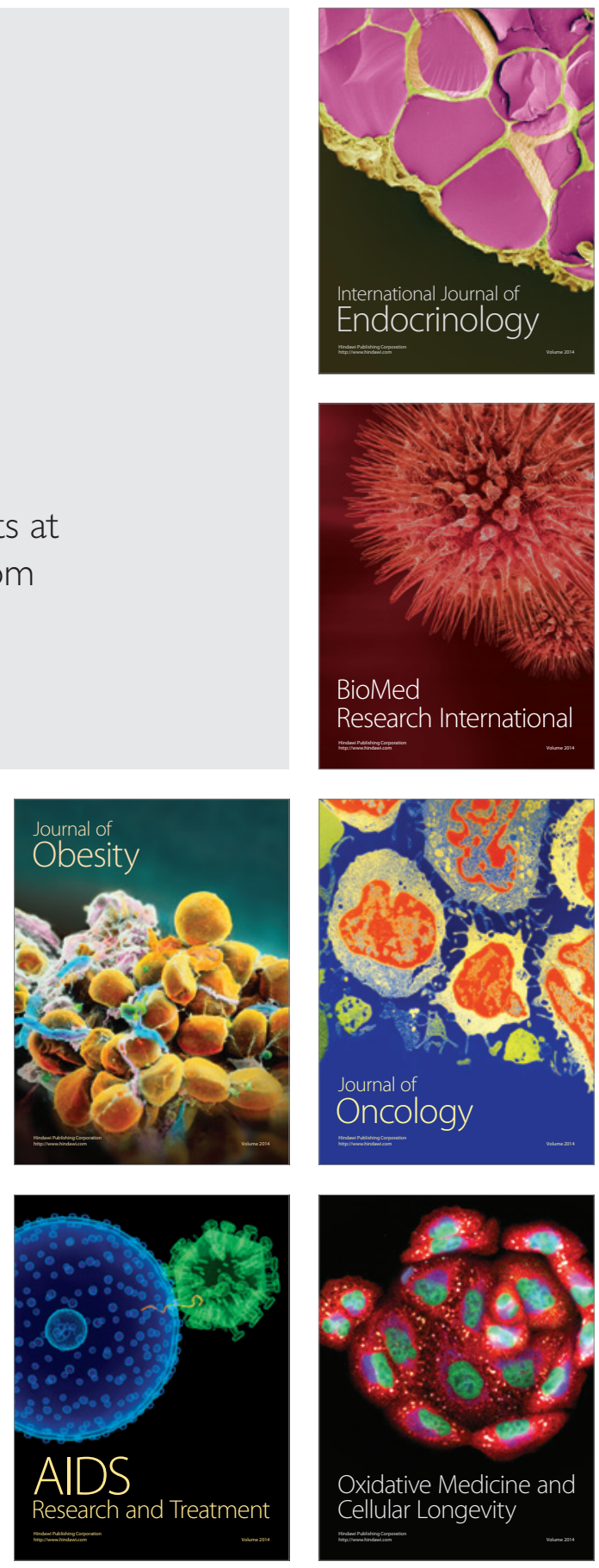